\title{
Central Pattern Generators (CPG) biomimétiques en temps-réel sur FPGA pour des expérimentations bio- hybrides
}

Real-time biomimetic Central Pattern Generators (CPG) in FPGA for biohybrid experiments

\author{
Matthieu Ambroise ${ }^{1}$, Sébastien Joucla ${ }^{2}$, Blaise Yvert $^{2,3}$, Sylvain Saïghi ${ }^{1}$, Timothée Levi ${ }^{1}$ \\ ${ }^{1}$ IMS Lab., Univ Bordeaux, Talence, matthieu.Ambroise@newcastle.ac.uk, sylvain.saighi@u-bordeaux.fr, \\ timothee.levi@u-bordeaux.fr \\ ${ }^{2}$ INCIA, Univ Bordeaux, Talence, joucla_sebastien@yahoo.fr \\ ${ }^{3}$ Inserm, BrainTech Lab U1205, Grenoble, blaise.yvert@inserm.fr \\ ${ }^{4}$ Univ Grenoble Alpes, BrainTech Lab U1205, Grenoble
}

RÉSUMÉ. L'hybridation est une technique qui consiste à interconnecter un réseau de neurones biologiques et un réseau de neurones artificiels. Elle est notamment utilisée dans la recherche en neuroscience et à des fins thérapeutiques. L'objectif à long-terme est de remplacer les réseaux de neurones endommagés par des systèmes artificiels. Ceux-ci requièrent le développement de modèles de neurones dont l'activité électrique est similaire à l'activité des réseaux biologiques vivants. Cette correspondance permet de produire une stimulation adéquate afin de restaurer la fonction neurale désirée.

Dans cet article, un réseau de neurones artificiels numériques avec une architecture configurable a été réalisé. Le réseau de neurones artificiels permet d'émuler l'activité de CPGs (Central Pattern Generator), à l'origine de la locomotion chez les animaux. Cette activité permet de déclencher une série de stimulations sur une moelle épinière lésée et de recréer ainsi la locomotion précédemment perdue. Ces résultats sont une première étape vers des solutions hybrides artificiel / biologique basées sur la micro-stimulation électrique pour la restauration de fonctions du Système Nerveux Central (SNC).

ABSTRACT. Hybridization is a technique that consists in interconnecting a network of biological neurons and a network of artificial neurons. It is used in neuroscience research and for therapeutic purposes. The long-term goal is to replace damaged neural networks by these artificial systems. These require the development of models of neurons whose electrical activity is similar to the activity of living biological networks. This correspondence allows to produce an adequate stimulation in order to restore the desired neural function. In this paper, digital artificial neural network with a configurable architecture has been designed. This network of artificial neurons emulates the activity of CPGs (Central Pattern Generator), at the origin of the locomotion. This activity triggers a series of stimulations on an injured spinal cord and thus recreates the previously locomotion. These results are a first step toward hybrid artificial/biological solutions based on electrical micro-stimulation for the restoration of lost function in the injured CNS (like locomotion).

MOTS-CLÉS. FPGA, réseau de neurones, Izhikevich, colonne vertébrale, expérimentation hybride.

KEYWORDS. FPGA, neural network, Izhikevich, spinal cord, hybrid experiment.

\section{Introduction}

Des millions de personnes à travers le monde sont affectées par des troubles neurologiques qui nuisent à la bonne communication entre le cerveau et le corps, causant différents handicaps (paralysies ou troubles des capacités cognitives). Ce nombre est prévu à la hausse pour les prochaines années tandis que la technologie assistive est toujours limitée. Durant la dernière décennie, beaucoup de travaux de recherche se sont dévoués aux interfaces cerveau-machine (ICM) et aux neuroprothèses en général [HOC06], [HOC12], [NIC09], dans le but de trouver un traitement efficace contre ces handicaps. Le développement de tels dispositifs a eu et continuera d'avoir un impact social sur la qualité de vie des patients. Ces prothèses sont conçues sur la base de nos connaissances des interactions des cellules neuronales, en partant des activités spontanées intrinsèques des réseaux de 
neurones jusqu'à la stimulation des réseaux de neurones afin d'obtenir un état ou comportement spécifique. L'objectif à long-terme de remplacer les réseaux de neurones endommagés par des systèmes artificiels requiert le développement de modèles de neurones dont l'activité est similaire à l'activité électrophysiologique des réseaux biologiques vivants. Cette correspondance permettra de produire une stimulation adéquate afin de restaurer la fonction neurale désirée. Dans notre étude, la configuration matérielle utilisée pour l'interface du composant biologique est un réseau de neurones artificiels (RNA) [LEV08], implémentant un modèle de neurone biologiquement réaliste. Ceci nous permettra d'émuler l'activité d'un réseau de neurones spécifique : un Central Pattern Generator (CPG).

L'objectif principal de cette étude est de créer un CPG artificiel qui sera connecté à une colonne vertébrale 'ex vivo' de rat et ainsi produire une locomotion fictive. Le but final du projet est le développement d'une nouvelle génération de neuroprothèses capable de rétablir les communications rompues entre différents réseaux de neurones localisés au sein de la colonne vertébrale, et ainsi rétablir la locomotion.

Plusieurs systèmes pionniers neuromorphiques abritant un RNA en vue d'expérimentations hybrides ont été développés [JUN01][LEM02] [VOG06]. Dans le domaine animal, la locomotion est une des capacités de base. Les neurobiologistes ont établi que la locomotion résulte de l'activité d'oscillateurs locomoteurs fournissant des bouffées de potentiels d'actions (PA) alternées. Le premier oscillateur de ce type a été observé et proposé par [BRO14], décrivant un réseau de neurone composé de neurones moteurs contrôlant la flexion et l'extension. La plupart des mouvements rythmiques est provoquée par les CPGs [MAR01], [IJS08]. Les CPGs [HOO00] sont des réseaux de neurones produisant un pattern rythmique de manière autonome. Ces oscillateurs locomoteurs contrôlent par exemple la nage chez la salamandre [IJS07] et la lamproie [COH92] et aussi le battement cardiaque chez la sangsue [CYM02]. Suivant la complexité de la fonction à remplir, les CPGs vont varier en taille (variation dans le nombre de neurones et de synapses). L'article au centre de notre étude du CPG est [HIL01], dans lequel le formalisme Hodgkin-Huxley a été utilisé pour reproduire le CPG à l'origine du battement de cœur chez la sangsue. Ce CPG est à la fois le plus simple et le plus petit CPG du domaine animal et cette particularité en fait un précieux point de départ pour notre étude de CPG en numérique.

La principale nouveauté de notre système est d'implémenter le réseau de neurones à l'origine du battement cardiaque chez la sangsue en utilisant un nombre de ressources minimum tout en maintenant une activité biologiquement réaliste. En effet, l'application finale est l'expérience hybride, c'est-à-dire une connexion entre un réseau de neurones artificiels (RNA) embarqué sur une plateforme FPGA (Field Programmable Gate Array) et des tissus biologiques (dans notre étude, une moelle épinière). Pour atteindre un tel objectif, notre plateforme FPGA, doit en plus du RNA, posséder des modules de détection de potentiel d'action (PA), de traitements de données biologiques et de stimulation par microélectrodes, en temps réel. Ceci explique notre contrainte supplémentaire dans la conception de notre RNA: la nécessité d'utiliser un minimum de ressources. Pour y arriver, le modèle de CPG et les résultats proposés par [HIL01] ont été reproduits en utilisant un formalisme plus simple (formalisme d'Izhikevich [IZH04]), implémenté dans une plateforme numérique équipée d'un FPGA. Cette plateforme permet un design léger (utilisant peu de ressources) d'un réseau de CPG temps réel. Dans notre cas, un réseau de 240 CPGs implémentés sur une plateforme FPGA Spartan6. Cette implémentation nous permet par ailleurs de reproduire l'activité présente chez la salamandre [IJS01] qui nécessite 40 CPGs ou de développer les expériences hybrides [LEM02] pour le développement de neuroprothèses nouvelle génération [BRA12] [HYR12].

La première partie de cet article décrit le réseau de neurones biologiques à l'origine du battement de cœur chez la sangsue. La partie suivante présente l'implémentation sur laquelle s'appuie le CPG artificiel. Ensuite, nous aborderons l'expérience hybride menée sur une moelle épinière de rat 'ex vivo'. 


\section{Topologie de l’implémentation matérielle du réseau de neurone}

\subsection{Etat de l'art}

Dans le règne animal, le mouvement (la marche chez la salamandre [IJS07], la nage chez les têtards [LI14], le vol chez les criquets [STE87] et même les battements de cœur [HIL01] [CYM02] sont contrôlés par des CPGs. [IJS08] a démontré que les implémentations de CPGs sont divisées selon différents niveaux d'abstraction. Ces niveaux commencent par l'approche biophysique du modèle Hodgkin-Huxley (HH) [HOD52], et aboutissent à une approche plus abstraite (l'approche mathématique), basée sur les oscillateurs couplés [ZIE96]. Entre ces deux extrêmes se trouve l'approche "connexionniste", utilisant des modèles simples de neurones impulsionnels pour étudier les mécanismes spécifiques aux CPGs [EKE93].

Le but de l'approche biophysique est d'atteindre une proximité (ou même une équivalence) entre le système biologique et le modèle simulé afin de faciliter ou rendre possible l'expérimentation hybride [BON13], [LEV 16]. C'est pourquoi [HIL01] rapporte les mesures effectuées sur un CPG biologique responsable du battement du cœur chez la sangsue avant d'utiliser le modèle HH pour émuler l'activité caractéristique de ce CPG. Comme nous l'avions évoqué, le modèle HH est peu adapté pour une implémentation numérique [LEV 17].

L'approche abstraite se focalise principalement sur la structure des CPGs et les dynamiques de leurs phases. La neurorobotique utilise les CPGs pour coordonner les mouvements de robots bipèdes [LI13], multipèdes [BAR13] ou robots nageurs [IJS07]. Dans le domaine de la neurorobotique, les CPGs sont implémentées à l'aide de microcontrôleurs ou microprocesseurs [CRE06], utilisant principalement des oscillateurs sinusoïdaux non-linéaires (Fitzhugh-Nagumo [FIT55] [FIT61] [NAG62], Stein [STE67], Van der Pol [VAN20] [VAN28]). Les dynamiques décrites par ces systèmes ont été étudiées en utilisant des outils mathématiques classiques [ZIE96] de manière à faciliter l'émulation des divers mouvements trouvés dans le règne animal (comme le trot, la marche et le galop [BAR13]). En dépit d'une possible implémentation du modèle Van Der Pol sur FPGA, démontré par [BAR13], le niveau d'abstraction est trop élevé pour envisager une connexion avec des organismes vivants.

L'approche connexionniste est nécessaire pour réaliser un CPG sur une plateforme FPGA. Elle se caractérise par l'utilisation d'un modèle de neurone impulsionnel utilisant peu de ressources et compatible pour l'expérimentation hybride. Le modèle Izhikevich (IZH) [IZH03] possède les qualités requises pour cette implémentation. Afin de pouvoir implémenter le premier CPG utilisant le modèle IZH, nous avons d'abord étudié les CPGs biologiques dans la littérature et passé en revue les différentes implémentations de CPGs. Nous allons présenter notre CPG, sa topologie et les paramètres utilisés, en rappelant que notre objectif est de s'approcher le plus possible des organismes vivants. C'est d'ailleurs pour cela que, dans la suite de cette étude, nous allons comparer l'activité de notre implémentation de CPG avec l'activité mesurée dans le système biologique.

\subsection{Description des CPGs biologiques}

Bien qu'utilisant un formalisme différent d'Izhikevich, [MAT87] présente plusieurs CPGs ayant différentes topologies. Par topologie, nous parlons de la structure du réseau de neurones c'est-à-dire, le nombre de neurones et le type de synapses qui les relient les uns aux autres. L'étude affirme alors que les CPGs nécessitent peu de neurones et décrit par conséquent des CPGs comprenant entre 2 et 6 neurones pour 2 à 18 synapses. De plus, [MAT87] et [HIL01] décrivent des CPGs comme des réseaux ayant une inhibition mutuelle. [HIL01] va plus loin dans son étude en présentant le CPG à l'aide du formalisme d'Hodgkin-Huxley produisant une activité semblable au CPG biologique à l'origine du battement de cœur chez la sangsue. Cette étude nous montre alors que le CPG élémentaire est composé de 2 neurones (proche de la description RS donnée par Izhikevich) reliés mutuellement par 2 synapses inhibitrices (figure 1.A). Ces études conjointes vont donc conditionner notre expérimentation, à savoir que notre CPG élémentaire sera composé de 2 neurones RS connectés par 2 synapses inhibitrices ayant 
une plasticité à court-terme. [HIL01] présente également un CPG segmental composé de 8 neurones et des synapses inhibitrices (figure 1.B). Nous allons donc dans un premier temps étudier le CPG élémentaire, puis dans un deuxième temps le CPG segmental pour notre expérimentation hybride.

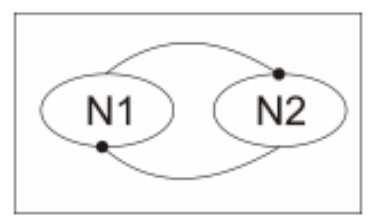

(a)

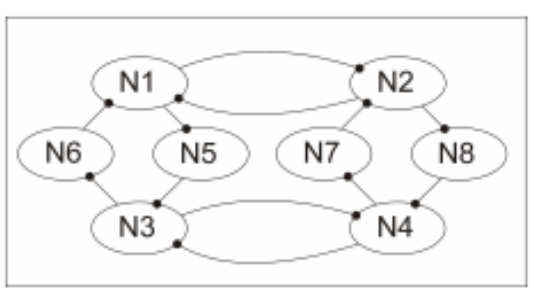

(b)

Figure 1. A) Topologie d'un oscillateur élémentaire B) Topologie d'un oscillateur segmental. Ces 2 réseaux sont composés de neurones $R S$ connectés par des synapses inhibitrices

\subsection{Choix et présentation du modèle de neurones d'Izhikevich}

Un modèle de neurone impusionnel ou formalisme est une description mathématique de certaines propriétés d'un neurone, créé pour imiter et prédire le comportement biologique d'un neurone. Ces modèles vont décrire les dynamiques régissant les variations de la tension de membrane. Parmi ceuxci, certains modèles décrivent le potentiel de membrane par une variable unique, en mettant de côté la dimension spatiale, et sont appelés modèle à compartiment simple. Dans ce groupe, beaucoup de dynamiques de neurones biologiques (figure 2) peuvent être reproduites grâce à des modèles de neurones reposant sur les conductances ioniques. Parmi ces modèles à conductances, se trouve le modèle Hodgkin-Huxley $(\mathrm{HH})$. Les modèles à compartiment simple permettent en effet de décrire avec précision les dynamiques de neurones et ainsi d'émuler le comportement de plusieurs familles de neurones. Cependant, ces modèles ne permettent pas de retranscrire d'autres réalités biologiques telle que la physiologie des dendrites ou des axones. Ces derniers sont intégrés dans d'autres modèles dits à compartiments multiples ou des modèles reposant sur la théorie des câbles. Cependant les modèles à compartiment unique sont capables de générer plusieurs familles de neurones.

Dans la conception d'un RNA, la première étape est le choix d'un modèle biologiquement réaliste. Notre choix de modèle a été basé sur deux critères: les familles des neurones pouvant être reproduites et le nombre d'équations le composant. Ces critères ont été utilisés pour comparer plusieurs modèles.

[HIL01] utilise le modèle HH pour reproduire le système de battements cardiaques avec huit neurones (figure 3.B). À partir des équations définies dans son article, il a été établi que les huit neurones à l'origine du battement cardiaque chez la sangsue s'apparentent à des neurones de type RS (Regular Spiking). Le modèle HH reproduit tous les types de neurones de manière très précise (timing et forme des pointes). Les principaux inconvénients résident dans le grand nombre de paramètres et les équations requises pour le mettre en oeuvre. En effet, le modèle HH est composé de neuf équations de courant dépendantes de la tension de membrane du neurone. Le modèle HH nécessite 32 paramètres pour un neurone RS [GRA11]. En outre, un neurone RS nécessite quatre canaux ioniques (dynamique des ions potassium et sodium, le courant de fuite et le potassium lent).

Le modèle IZH représente une bonne solution, car il repose sur deux équations et est capable de reproduire de nombreuses familles différentes de neurones en changeant quatre paramètres. De plus, selon [IZH04], l'implémentation de ce modèle nécessite peu de ressources. Ceci représente un avantage clé dans notre objectif de conception d'un grand réseau de CPG sur une plateforme FPGA où résident d'autres modules nécessaires pour des expériences hybrides (détection et tri de potentiel d'action, stimulation, etc.).. Le modèle IZH dépend de quatre paramètres, ce qui le rend capable de reproduire le comportement de nombreux types de neurones corticaux dont le type RS. D'un point de 
vue mathématique, le modèle est décrit par un système bidimensionnel d'équations différentielles [IZH03]:

$$
\begin{aligned}
& \mathrm{v}^{\prime}=0.04 \mathrm{v}+5 \mathrm{v}+140-\mathrm{u}+\mathrm{I} \\
& \mathrm{u}^{\prime}=\mathrm{a} .(\mathrm{b} \cdot \mathrm{v}-\mathrm{u})
\end{aligned}
$$

Si la tension de membrane v est supérieure ou égale à $30 \mathrm{mV}$ (tension de seuil pour l'émission d'un PA), nous avons :

$$
\begin{aligned}
& \mathrm{v} \leftarrow \mathrm{c} \\
& \mathrm{u} \leftarrow \mathrm{u}+\mathrm{d}
\end{aligned}
$$

Dans l'équation (3), $v$ est le potentiel membranaire du neurone, $u$ est la variable de régénération de la membrane, qui prend en compte l'activation du potassium et l'inactivation des canaux de sodium. Le modèle IZH a été choisi pour imiter le comportement des cellules excitatrices pour sa simplicité et sa capacité à mettre en œuvre diverses familles de neurones [AMB13].

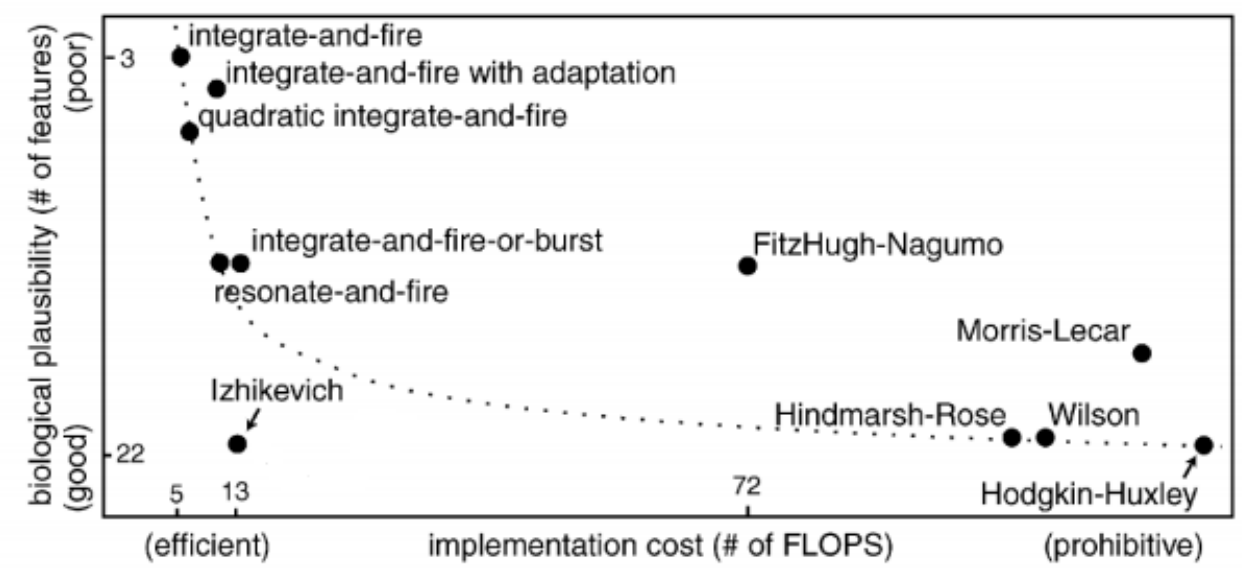

Figure 2. Coût de l'implémentation (mesuré en nombre d'opérations) en fonction du réalisme biologique du modèle de neurone (mesuré par le nombre de familles émulables). Cette figure a été copiée de [IZH04].

\subsection{Modèle de synapse}

\subsubsection{Plasticité à court terme}

Utiliser le modèle de synapse proposé dans [IZH04] nous permet de reproduire les mécanismes de dépression ou de facilitation d'une synapse selon lequel lorsqu'un neurone pré-synaptique émet un potentiel d'action, le courant synaptique associé au neurone post-synaptique est pondéré par le poids de la synapse liant le neurone pré-synaptique et le neurone post-synaptique. La plasticité à court-terme permet d'ajouter des dynamiques à notre réseau qui le rendront plus réaliste biologiquement parlant et permettra aussi la reproduction de l'activité des CPGs. Ce modèle est défini par 3 paramètres :

- un facteur scalaire xsyn qui va indiquer l'état de la synapse (dépression ou facilitation), qui pondère le poids synaptique et dont la valeur tend toujours vers 1 .

- un pourcentage $\mathrm{P}$ qui sera multiplié par le facteur xsyn à chaque émission d'un potentiel d'action pré-synaptique. Si ce pourcentage est supérieur à 1 , alors cette synapse décrira une facilitation à courtterme. Dans le cas contraire, si ce pourcentage est inférieur à 1 , cette synapse décrira une dépression à court-terme. Dans le cadre d'une facilitation (ou d'une dépression), la valeur ajoutée au courant de 
stimulation d'un neurone post-synaptique augmentera (ou diminuera) à chaque émission de potentiel d'action.

- une constante de temps tsyn de décroissance (ou croissance) exponentielle dans le cas d'une facilitation (ou dépression). Ce modèle s'appelle "plasticité à court-terme » car la facilitation comme la dépression d'une synapse se résorbe lorsqu'aucun potentiel d'action n'a pas été émis pendant un temps tsyn.

Notre cœur de calcul de synapse aura lui aussi 2 comportements différents si un potentiel d'action pré-synaptique est présent ou non, et sera donc régi par les équations présentées dans le tableau 1.1.

\begin{tabular}{|c|c|}
\hline $\begin{array}{c}\text { Lorsqu'aucun potentiel } \\
\text { d'action pré-synaptique a été } \\
\text { émis }\end{array}$ & $\begin{array}{c}\text { Lorsqu'aucun potentiel } \\
\text { d'action pré-synaptique n'a } \\
\text { pas été émis }\end{array}$ \\
\hline $\begin{array}{c}\mathrm{I}_{\mathrm{syn}}[\mathrm{n}+1]=\mathrm{I}_{\mathrm{syn}}[\mathrm{n}]+\mathrm{W}_{\mathrm{s}}[\mathrm{n}] \\
\text { Avec } \mathrm{W}_{\mathrm{s}}[\mathrm{n}]=\mathrm{x}_{\mathrm{syn}}[\mathrm{n}] \cdot \mathrm{w}_{\mathrm{syn}}\end{array}$ & $\mathrm{I}_{\mathrm{syn}}[\mathrm{n}+1]=\mathrm{I}_{\mathrm{syn}}[\mathrm{n}]$ \\
\hline $\mathrm{x}_{\mathrm{syn}}[\mathrm{n}+1]=\mathrm{P} \cdot \mathrm{x}_{\mathrm{syn}}[\mathrm{n}]$ & $\mathrm{x}_{\mathrm{syn}}[\mathrm{n}+1]=\mathrm{x}_{\mathrm{syn}}[\mathrm{n}]+\left(1-\mathrm{x}_{\mathrm{syn}}[\mathrm{n}]\right)$ \\
\hline
\end{tabular}

Tableau 1.1. Système d'équations régissant le cœur de calcul des synapses. Ce système prend en compte 2 cas, soit le neurone pré-synaptique a émis un potentiel d'action soit aucun potentiel d'action n'a été émis.

\subsubsection{Effet AMPA/GABA -ergique}

Pour approcher un comportement biologiquement réaliste, nous avons implementé l'effet AMPAergique et GABA-ergique. Il s'agit d'une décroissance exponentielle des courants synaptiques respectivement liés aux excitations et aux inhibitions. Des observations biologiques montrent que les excitations (ou inhibitions) subies par un neurone tendent vers zéro de manière exponentielle suivant une constante de $\tau$ exc(ou $\tau$ inh) [BEN97].

\subsection{Architecture globale du réseau}

En prenant l'exemple de [CAS08], il est possible de prendre le modèle IZH et de créer ce que nous appellerons un cœur de calcul. En effet, l'idée est de créer un module qui a pour tâche de calculer et mettre à jour les valeurs $u$ et $\mathrm{v}$ de chaque neurone composant notre réseau. De cette manière, [CAS08] nous livre une architecture multiplexé temporellement qui va donc calculer les valeurs $\mathrm{u}$ et $\mathrm{v}$ de chaque neurone en utilisant $\mathrm{Cn}$ cycles. Cela signifie que cette architecture apporte les coefficients d'Izhikevich $(\mathrm{a}, \mathrm{b}, \mathrm{c}$ et $\mathrm{d})$ et les variables d'état $\mathrm{u}$ et $\mathrm{v}$ d'un neurone $\mathrm{N}$ à un temps donné et le cœur de calcul de neurone donnera le résultat $\mathrm{Cn}$ cycles plus tard. Deux stratégies se révèlent possibles : une architecture multiplexée temporellement et une architecture pipelinée. Dans une architecture multiplexée, mettre à jour les 100 neurones de notre réseau requerra Cn cycles par neurone, pour un total de $100 \mathrm{x} \mathrm{Cn}$. D'un autre côté, une architecture pipelinée (architecture présentée dans ce travail de thèse) permet de faire les mêmes calculs pour les 100 neurones en $(100+\mathrm{Cn})$ cycles. Intuitivement, faire les calculs en moins de temps nous donne accès à un plus grand nombre de neurones.

L'architecture pipelinée est aussi appliquée aux synapses et ainsi nous pouvons décrire un neurone par ses paramètres ( $\mathrm{a}, \mathrm{b}, \mathrm{c}, \mathrm{d}, \mathrm{u}, \mathrm{v}$ et ses 3 courants), une synapse par les paramètres de plasticité ( $\mathrm{x}$, $\tau$ syn, p,Wsyn), les courants synaptiques et le PA du neurone pré-synatique. L'ensemble de ces paramètres sont stocké dans la mémoire RAM embarquée et seront redistribués lors des différentes phases de calculs. 
Il est à noter que d'autres modules ont été implémentés au sein du RNA, que nous n'avons pas étayé dans cet article, car ils n'interviennent pas dans la conception du CPG numérique comme le générateur de bruit par le processus d'Orhnstein-Ulhenbeck et le retard synaptique [AMB15], [GRA17].

\subsection{Validation par comparaison avec le comportement biologique}

Nous comparons tout d'abord l'activité biologique avec le comportement de notre RNA (figure 3).

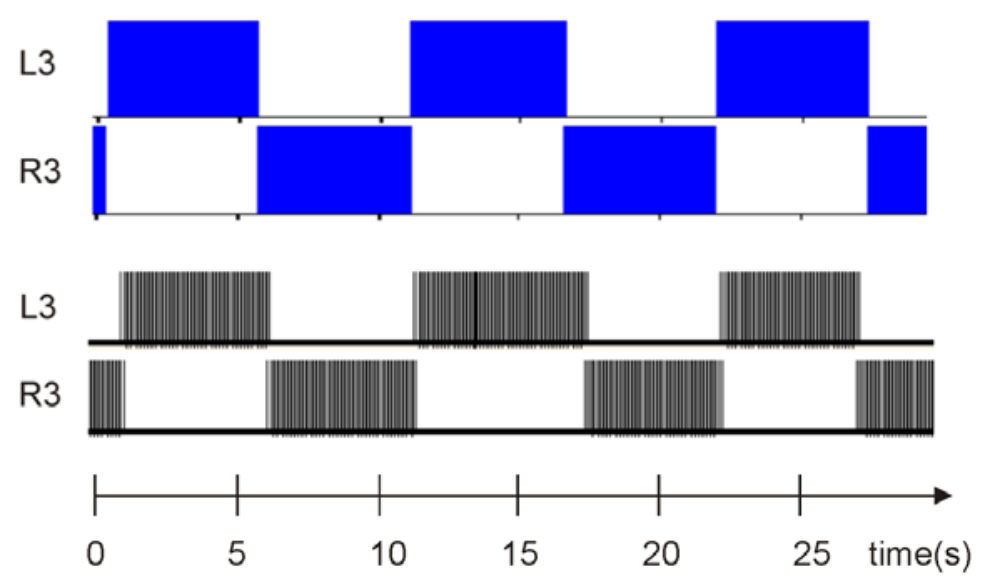

Figure 3. Comparaison de l'activité du CPG biologique (en bleu) et celui matériel (en noir). Le rapport cyclique et la fréquence sont identiques

\begin{tabular}{|c|c|c|c|}
\hline & $\begin{array}{c}\text { Système } \\
\text { biologique }\end{array}$ & $\begin{array}{c}\text { Oscillateur } \\
\text { numérique } \\
\text { élémentaire }\end{array}$ & $\begin{array}{c}\text { Oscillateur } \\
\text { numérique } \\
\text { segmental }\end{array}$ \\
\hline $\begin{array}{c}\text { moyenne } \\
\text { Rapport } \\
\text { cyclique moyen }\end{array}$ & $\begin{array}{c}57.2 \pm 2.9 \\
\%\end{array}$ & $49.2-50 \%$ & $56.5 \pm 4 \%$ \\
\hline $\begin{array}{c}\text { Fréquence } \\
\text { moyenne intra- } \\
\text { bouffée }\end{array}$ & $\begin{array}{c}11.9 \pm 2.1 \\
\mathrm{~Hz}\end{array}$ & $8.7 \pm 2.7 \mathrm{~Hz}$ & $9 \pm 2 \mathrm{~Hz}$ \\
\hline $\begin{array}{c}\text { Fréquence } \\
\text { initiale } \\
\text { moyenne }\end{array}$ & $\begin{array}{c}4.3 \pm 0.7 \\
\mathrm{~Hz}\end{array}$ & $6.1 \pm 0.2 \mathrm{~Hz}$ & $6.7 \pm 0.2$ \\
\hline $\begin{array}{c}\text { Fréquence } \\
\text { finale moyenne }\end{array}$ & $5.81 \pm \mathrm{Hz}$ & $8.3 \pm 0.4 \mathrm{~Hz}$ & $9 \pm 0.5 \mathrm{~Hz}$ \\
\hline
\end{tabular}

Tableau 1.2. Comparaison entre les oscillateurs numériques et le système biologique

Il est possible de changer la période des bouffées de PA du CPG de $1.3 \mathrm{~s}$ à $10 \mathrm{~s}$ en changeant les paramètres adéquats (tableau 1.3). 


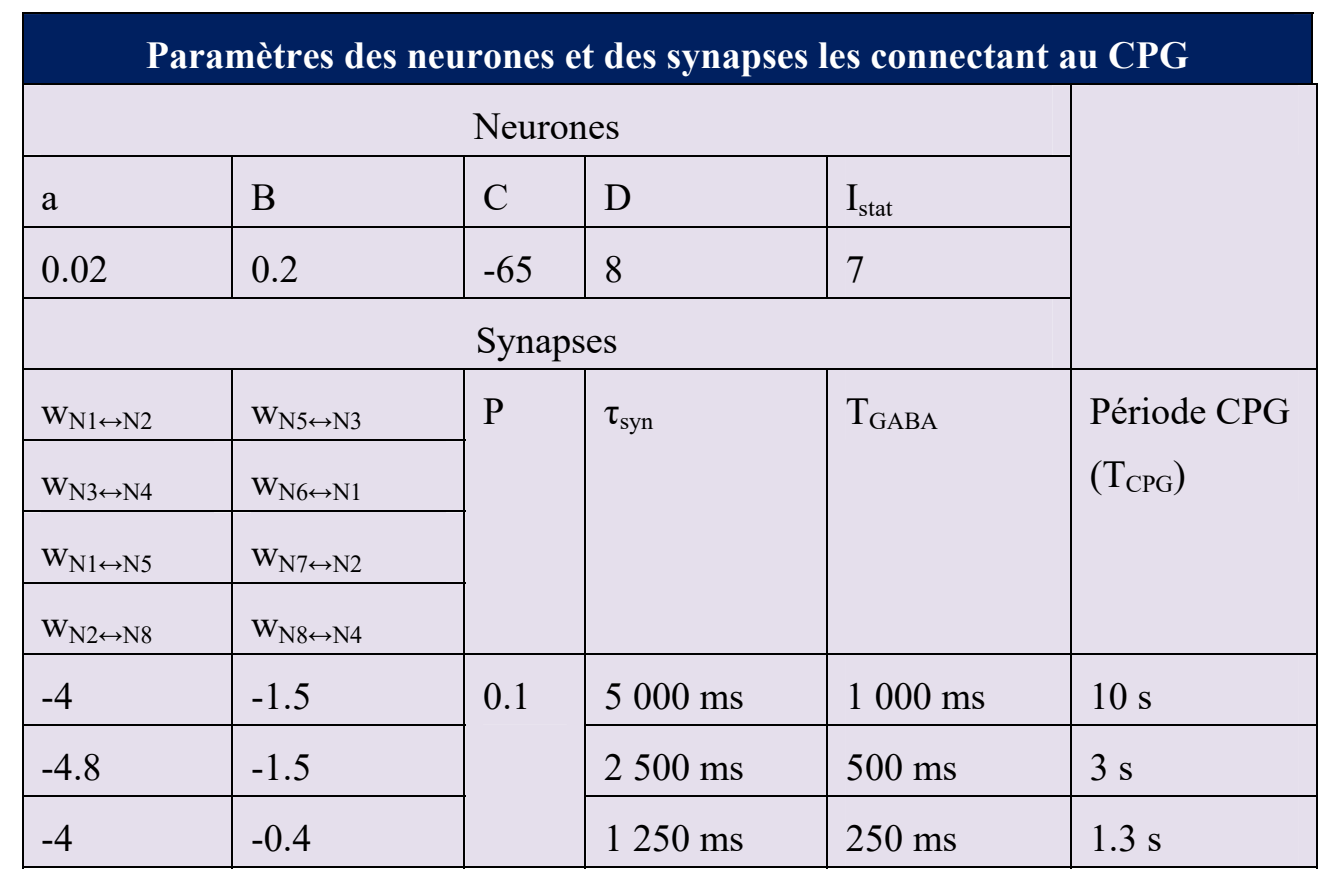

Tableau 1.3. Evolution de la période au sein de l'oscillateur segmental en fonction des paramètres du RNA (poids synaptiques, GABA et $T_{\text {syn }}$ )

Notre approche connexionniste nous permet d'obtenir une période moyenne et un rapport cyclique proche de la biologie. Ceci nous permet de déclencher les stimuli électriques avec un timing compatible avec le vivant. Cette validation nous permet de passer à l'étape suivant à savoir effectuer des expériences hybrides sur la moelle épinière 'ex vivo' d'un rat.

\section{Expérience hybride}

Les neuroprothèses axées sur la micro-stimulation électrique offrent des perspectives prometteuses pour restaurer les fonctions suite à des lésions du système nerveux central (SNC). Elles nécessitent l'identification des sites de stimulation appropriés et la coordination de leur activation pour réaliser la restauration de l'activité fonctionnelle. A long terme, une perspective est de contrôler la microstimulation par des réseaux de neurones artificiels hybridés avec le tissu vivant. En ce qui concerne l'utilisation de cette stratégie pour rétablir l'activité locomotrice dans la moelle épinière, il n'y a pas eu, à ce jour, de preuve de principe d'une telle approche hybride de contrôle de micro-stimulation intrarachidienne utilisant des réseaux de neurones artificiels. [CAP16] présente des travaux prometteurs dans ce sens sur des primates. Dans ce paragraphe, nous abordons une première étape vers cet objectif avec la moelle épinière de rat néonatal isolée 'ex vivo', qui permet d'afficher l'activité locomotrice, tout en offrant un accès facile aux circuits intrarachidiens. Des réseaux de microélectrodes ont été insérés dans la région lombaire pour déterminer les sites de stimulation appropriés. Nous avons alors utilisé notre CPG biomimétique pour générer une activité rythmique et qui a été hybridée à la moelle épinière vivante pour générer des micro-stimulations électriques sur les deux sites identifiés. En utilisant cette stratégie, des activités de locomotion sur les racines ventrales L2 / L5 bilatérales peuvent être générées soit dans la moelle épinière intacte soit dans celle sectionnée. Ces résultats sont une première étape vers des solutions artificiel / biologique hybrides pour la restauration de la fonction perdue dans le système nerveux central endommagé.

\subsection{Méthodes}

\subsubsection{Traitements de données}

Les signaux bruts de la racine ventrale ont été traités de la manière suivante pour extraire l'activité locomotrice (voir aussi [HEI12]): pour chaque échantillon de données, une moyenne mobile du signal 
calculé sur une fenêtre de $10 \mathrm{~ms}$ centrée sur cet échantillon a d'abord été soustraite. Les données brutes (suppression de DC), puis la valeur obtenue a été remplacée par la moyenne du signal calculé sur une fenêtre de temps de $1 \mathrm{~ms}$ centrée sur cet échantillon (lissage). Les signaux ont ensuite été intégrés pour évaluer les modèles alternatifs en utilisant des représentations polaires et des statistiques. À cette fin, les signaux ont d'abord été bloqués sur une période allant de $15 \mathrm{~ms}$ avant la stimulation à $150 \mathrm{~ms}$ après, puis intégrés à une constante de temps de $0,2 \mathrm{~s}$ (voir traces grises superposées à l'activité de pointage sur la figure 4.B) et enfin lissées avec une fenêtre de $1 \mathrm{~s}$. La signification statistique de la relation de phase entre l'activité d'éclatement enregistrée sur deux racines ventrales différentes a été évaluée à l'aide d'un test Rayleigh sur les retards entre les pics des deux signaux intégrés normalisés à $360^{\circ}$. La figure 4.C montre la représentation polaire typique d'une activité de type locomoteur induite pharmacologiquement, avec un verrouillage antiphase entre les racines ventrales L2 et L5 de chaque côté et les paires L2 ou L5 gauche-droite. Les méthodes d'analyses statistiques sont présentées dans [JOU16].

\subsubsection{Stimulation électrique}

Comme le montrent les figures 4.D-F, des micro-stimulations électriques ont été délivrées sur des microélectrodes individuelles de sondes neuronales Neuronexus constituées de quatre tiges séparées par $400 \mu \mathrm{m}$, chacune contenant huit microélectrodes de $30 \mu \mathrm{m}$ de diamètre séparées par $100 \mu \mathrm{m}$. Sur la base d'études antérieures montrant la localisation des CPG de rat [CAZ95] [ANT11], la sonde neurale a été insérée au niveau L1 de la moelle épinière. Selon la préparation, entre 15 et 26 sites de la sonde ont été testés individuellement pour la stimulation. Chaque stimulation consistait en un train de 10 impulsions biphasiques séparées par $1 \mathrm{~ms}$. Chaque impulsion a été équilibrée en charge avec une phase cathodique initiale de $500 \mu$ s et une amplitude typiquement de 150 à $300 \mu \mathrm{A}$, immédiatement suivie d'une phase anodique 10 fois plus longue d'amplitude 10 fois plus petite. Les stimulations étaient monopolaires par rapport à une électrode éloignée dans le bain.

\subsubsection{Hybridation: Du CPG artificiel vers la colonne vertébrale}

Les 8 neurones du CPG possèdent les mêmes paramètres (tableau 1.3). Nous avons divisé ces 8 neurones en différents groupes gauche (N1L à N4L) et droite (N1R à N4R) (voir figure 4.H). Ces neurones produisent une activité robuste en bouffée semblable à celle décrite dans la figure 4.I. Pour finir, nous ajoutons 2 neurones de sortie (paire de neurones N5L et N5R) qui généreront à leur tour un PA pour chaque bouffée générée par la paire N4L/N4R.

Ainsi les neurones N5L, N5R du CPG produisent une activité rythmique alternée gauche/droite avec un potentiel d'action par cycle de burst sur chaque côté du réseau. Ces potentiels d'actions de sortie sont utilisés comme signal de déclenchement pour les microstimulations électriques sur deux microélectrodes, une de chaque côté de la moelle épinière. Ainsi, la stimulation n'est déclenchée que par l'activité du CPG et se compose d'un train court de 10 stimuli. En changeant les paramètres adéquats, la période d'alternance de CPG artificielle est modulée pour des périodes fixes allant de $2.6 \mathrm{~s}$ à $6.7 \mathrm{~s}$.

\subsection{Génération d'une activité locomotrice dans une colonne vertébrale ex vivo utilisant une connexion hybride}

Dans un premier temps, nous avons créé une connexion hybride entre le CPG artificiel et la colonne vertébrale. La sortie des 2 neurones N5 du CPG artificiel contrôle les stimulations intraspinales. Chaque potentiel d'action du neurone de sortie N5 déclenche une stimulation sur le site intra-moelle correspondant. En utilisant cette stratégie, nous obtenons une activité motrice sur les racines ventrales bilatérales L2 et L5. Une fois que le CPG artificiel était actif, l'activité locomotrice au sein de la colonne vertébrale a été établie dès la première ou deuxième stimulation. L'activité ainsi engendrée est restée robuste avec une correspondance 1:1 au rythme artificiellement imposé tant que le CPG artificiel était activé, puis disparu dès que le CPG a été éteint. 


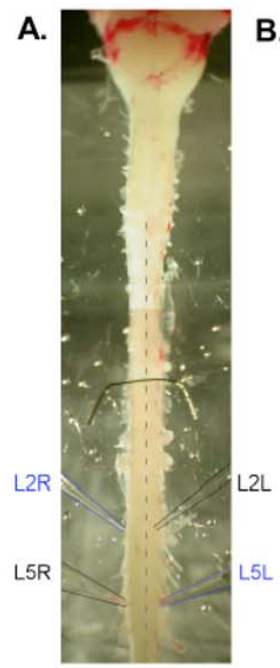

D.

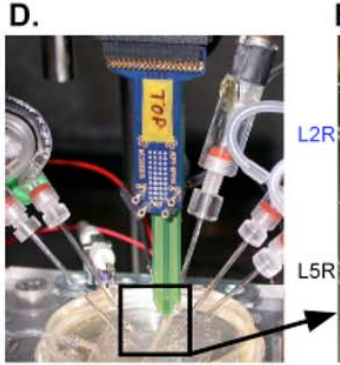

B. $L 5 L$

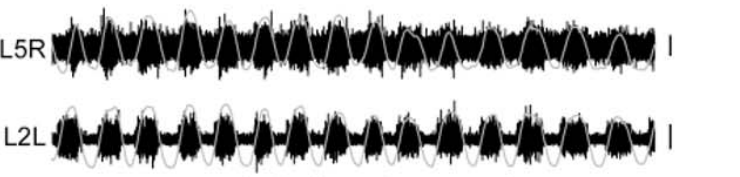

L2R M
C.

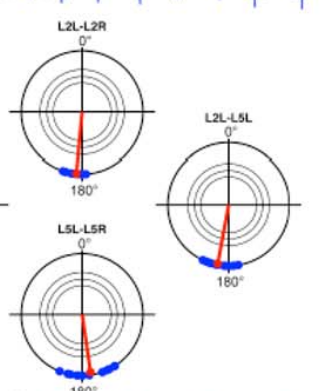

E.
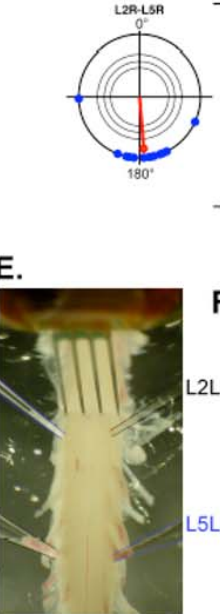

$5 \overline{\mathrm{sec}}$

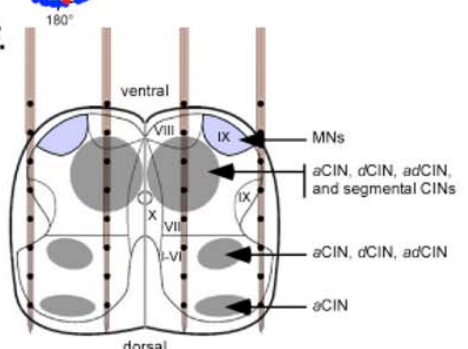

G.

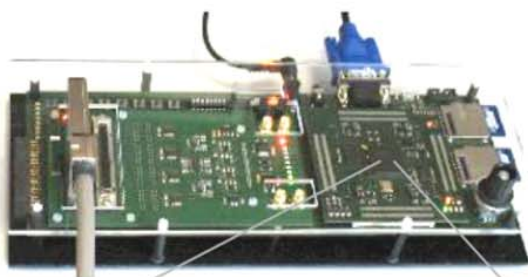

H.

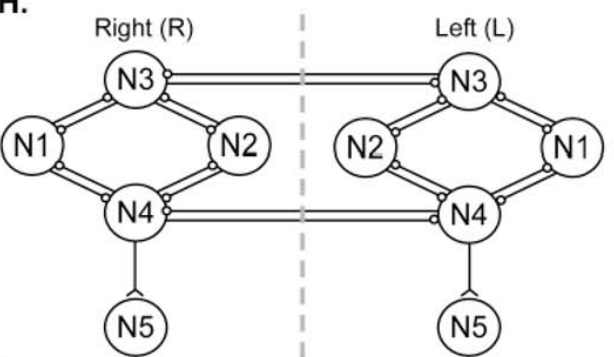

I.

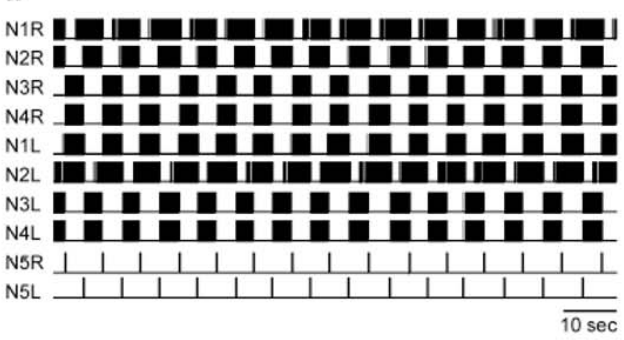

Figure 4. (A) Préparation de la moelle épinière 'ex vivo' du rat néonatal. (B) Exemple d'activité locomotrice sur les racines ventrales $L 2$ et $L 5$ bilatérales. (C) Représentation polaire du rythme entre les différentes racines

$(L 2, L 5)$ et entre les côtés $(L-R)$. ( $D, E)$ Description de l'installation expérimentale des enregistrements de racines ventrales et d'une sonde neurale multicanaux à 4 broches insérée au niveau L1 pour la microstimulation. (F) Représentation schématique de la sonde neurale dans le plan transversal de la moelle épinière adapté de [KIE03]. MNs : Motoneurones; CINs : interneurones commissurales. (G) Vue de la plate-

forme hardware qui héberge le CPG artificiel implémenté sur FPGA. (H) Structure du CPG artificiel (les connexions "o" sont inhibitrices, "<" les connexions sont excitatrices). (I) Exemple d'activité de tous les neurones du CPG artificiel. Les neurones N3R-N3L et N4R-N4L représentent les deux CPGs, N5L et N5R ont été utilisés pour déclencher la micro-stimulation [JOU16].

\subsection{Génération d'une activité locomotrice dans une colonne vertébrale sectionnée transversalement en utilisant une connexion hybride}

Dans un deuxième temps, nous avons alors considéré une autre préparation dans laquelle la colonne a été sectionnée au niveau T7 (figure 5.A). Le même type de connexion hybride a été utilisé entre le CPG artificiel et les sites de stimulation intra-moelle. Cette stratégie a aussi déclenché avec succès une activité locomotrice aux racines ventrales L2 et L5. Le pattern locomoteur peut être maintenu pendant plus de 7 minutes tant que le CPG artificiel est actif. De plus, plusieurs périodes d'alternance du CPG artificiel ont été testées dans cette préparation $(2.6,3.1,4,5.2$ et $6.7 \mathrm{~s}$ entre les stimulations successives gauche/droite). Nous avons remarqué que la colonne vertébrale suivait le rythme imposé avec une exacte correspondance 1:1 aux différentes fréquences pendant toutes les différentes durées de tests des connexions hybrides : $560 \mathrm{~s}$ avec un CPG de période $6.7 \mathrm{~s}$ (84 stimulations gauche/droite), $430 \mathrm{~s}$ avec un CPG de période $5.2 \mathrm{~s}$ ( 85 stimulations), $369 \mathrm{~s}$ avec un CPG de période $4 \mathrm{~s}$ (97 stimulations), $392 \mathrm{~s}$ avec un CPG de période $3.1 \mathrm{~s}$ (123 stimulations), and $363 \mathrm{~s}$ avec un CPG de période $2.6 \mathrm{~s}$ (217 stimulations). Le CPG artificiel était limité à ces périodes et nous n'avons pas pu tester de vitesses supérieures afin de déterminer quand la colonne vertébrale aurait arrêté de suivre le rythme imposé par le CPG artificiel. Néanmoins, les fréquences testées ont couvert le rythme pharmacologiquement 
évoqué avec une période d'inter-bouffées autour de $5 \mathrm{~s}$ sur chaque racine ventrale (comme le montre la figure 5.B).
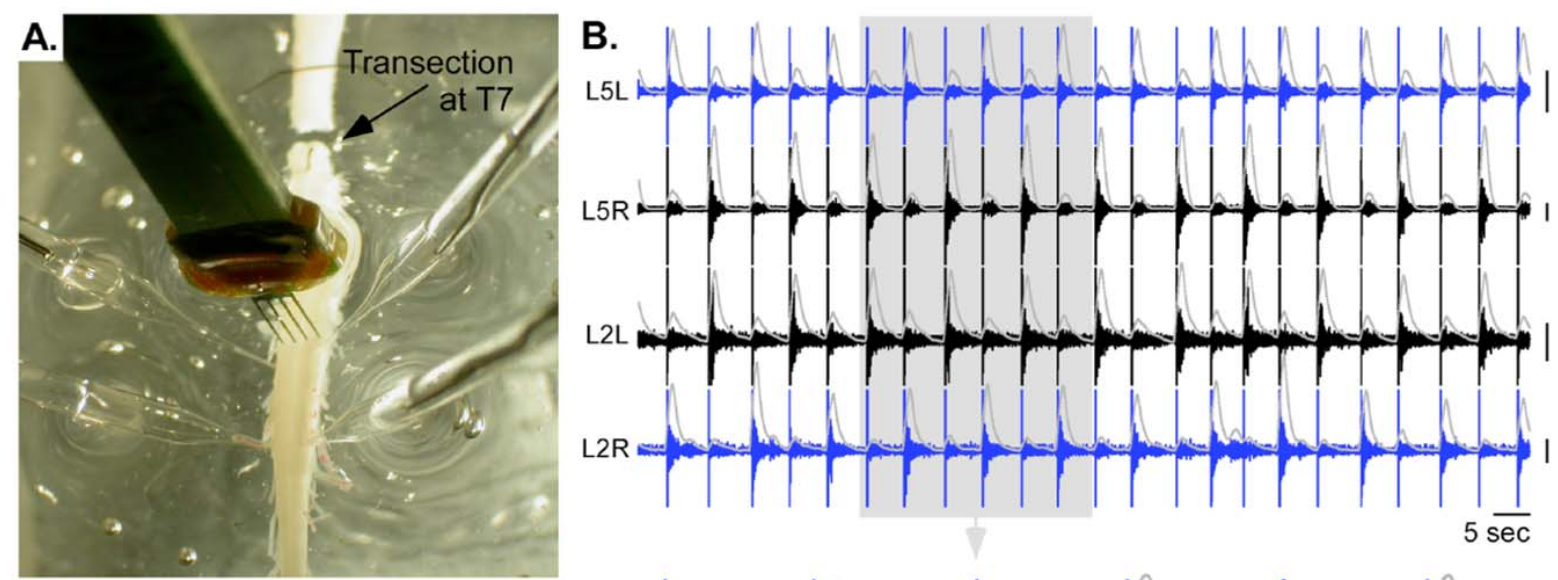

C.
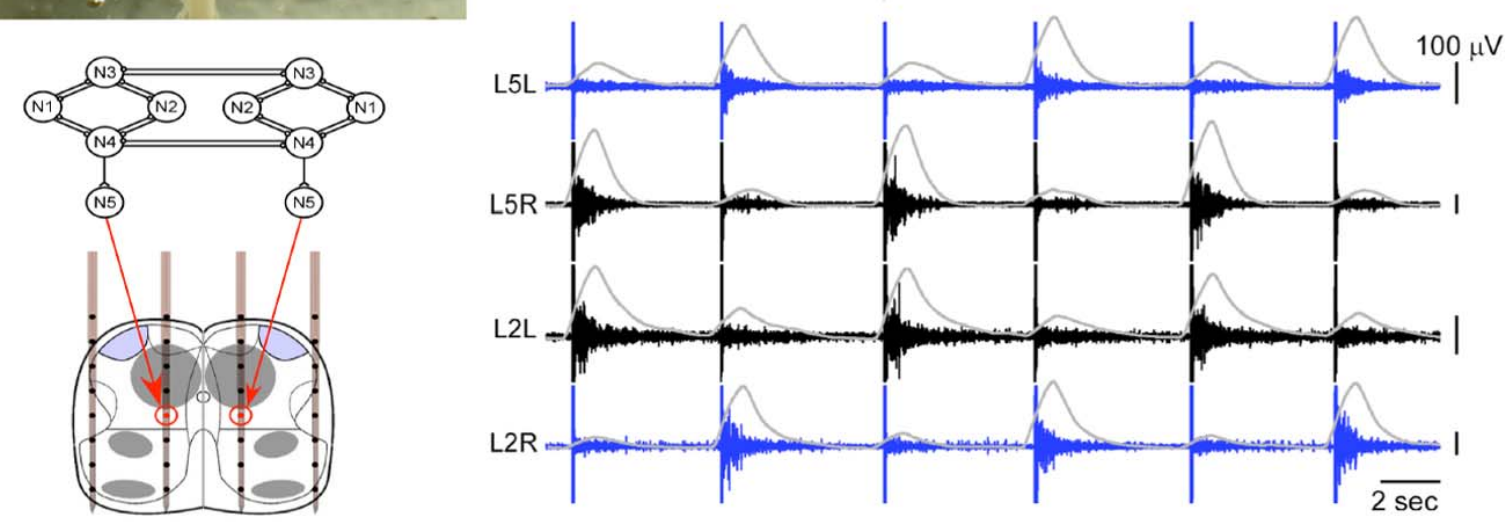

Figure 5. (A) Photos de moelle épinière de rat utilisé lors des expérimentations hybrides. Nous voyons la lésion au niveau T7, l'électrode en fourchette pour la stimulation et les sondes d'enregistrements. (B)

Enregistrements de l'activité des racines ventrales $L 2 / L 5$ suite aux stimulations pilotées par les $P A s$ des neurones NR et NL. (C) Schéma décrivant la connexion hybride entre les sorties du CPG artificiel et les deux sites de stimulation intraspinale.

La figure 5.B nous montre un exemple où les neurones NR/NL du CPG artificiel émettent des PAs alternés d'une période moyenne de 10 secondes. Ces PAs sont injectés dans le stimulateur qui va, à son tour, provoquer une micro-stimulation dans les racines L2 et L5. Nous constatons alors que les racines rendues inactives par la lésion de la moelle épinière reprennent une activité en bouffée cadencée par les PAs des neurones NR/NL.

\section{Conclusion}

Nous avons developpé un réseau de neurones artificiels biomimétiques afin de reproduire l'activité des CPGs biologiques. Nous avons validé ce sytème avec des experiences hybrides sur la moelle épinière de rat. Ces résultats sont une première démonstration d'une interconnexion hybride entre une moelle épinière 'ex vivo' et un réseau neuronal artificiel biomimétique controllant la microstimulation interspinale afin de restaurer une activité fonctionnelle de locomotion. Cette recherche mène vers des prothèses neurales intelligentes basées sur des connexions hybrides artificiel / vivant pour la restauration de fonctions du système central nerveux.

La quête d'une prothèse neurale entièrement autonome basée sur des connexions hybrides entre le $\mathrm{SNC}$ et les réseaux neuronaux artificiels est un énorme défi. Ici, nous n'avons effectué qu'une première étape vers cet objectif en établissant une connexion unidirectionnelle (boucle ouverte) entre un CPG artificiel et le circuit de la moelle épinière. Dans ce travail, le réseau artificiel a d'abord été configuré avec des paramètres appropriés pour présenter une activité rythmique en alternance adéquate. En 
particulier, la modification de la fréquence du rythme nécessite une reconfiguration (manuelle et une nouvelle synthèse du réseau dans le FPGA). D'autres versions de l'implémentation matérielle du CPG offriront la possibilité de moduler sa dynamique en temps réel. Cela ouvrira alors la possibilité de construire des connexions hybrides bidirectionnelles [AMB17], où l'activité supralésionnelle peut être utilisée pour contrôler dynamiquement le réseau artificiel afin d'obtenir une connexion artificielle en boucle fermée sur la lésion. En particulier, les futurs développements pourraient inclure des paradigmes où le réseau artificiel serait dynamiquement contrôlé et / ou modulé par des entrées provenant d'informations supralésionnelles et éventuellement modulés en temps réel par des réactions sensorielles produites après la microstimulation intraspinale dans des préparations préservant les membres postérieurs entiers attachés à la moelle épinière.

Dans une perspective supplémentaire, une telle approche hybride nécessitera également d'être prolongé 'in vivo' afin d'évaluer s'il peut aussi aider à récupérer les capacités de locomotion chez les animaux adultes sujets aux lésions chroniques de la moelle épinière. Des recherches ont été menées sur la colonne vertebrale de chiens [GUE06] [MAZ12], des résultats ont déjà été obtenu chez les rats [SHA14] et chez les primates [CAP16].

\section{Bibliographie}

[AMB13] Ambroise M., LeVI T., Joucla S., YVert B, SAÏGHI S., « Real-time biomimetic Central Pattern Generators in an FPGA for hybrid experiments », Frontiers in Neurosciences, 7:215, November 2013.

[AMB15] AMBroISE M., «Hybridation des réseaux de neurones : De la conception du réseau à l'interopérabilité des systèmes neuromorphiques», Thèse de doctorat, Université de Bordeaux, 2015.

[AMB17] Ambroise M, Buccelli S, Grassia F, Pirog A, Bornat Y, Chiappalone M, Levi T, « Biomimetic neural network for modifying biological dynamics during hybrid experiments », Journal on Artificial Life and Robotics, 22:3, 398-403, September 2017.

[ANT11] ANTRI, M., Mellen, N., AND CAZAlEts, J. R., «Functional organization of locomotor interneurons in the ventral lumbar spinal cord of the newborn rat», PLoS ONE 6:e20529. doi: 10.1371/journal.pone.0020529, 2011.

[BAR13] BARRON-ZAMBRANO, J. H., AND TORRES-HUITZIL, C., «FPGA implementation of a configurable neuromorphic CPG-based locomotion controller», Neural Netw.45, 50-61. doi: 10.1016/j.neunet.2013.04.005, 2013.

[BEN97] Ben-Ari, Y., KhaZipov, R., Leinekugel, X., CAIllard, O., AND Gaiarsa, J. L., «GABA A, NMDA and AMPA receptors: a developmentally regulated 'ménage à trois'», Trends Neuros c i.20, 523-529. doi: 10.1016/S01662236(97)01147-8, 1997.

[BON12] Bonnet, S., BÊCHE, J.-F., GHARBI, S., ABdoun, O., BoCQuelet, F., Joucla, S., ET AL., «A real-time multielectrode array system for recording, processing and stimulation of neural networks and the control of high-resolution neural implants for rehabilitation», IRBM 33, 55-60. doi: 10.1016/j.irbm.2012.01.013, 2012.

[BOn13] Bonifazi P., Difato F., Massobrio P., Breschi Gl., Pasquale V., LeVi T., Goldin M., Bornat Y., TEDesco M., Bisio M., KANNER S., GAlRON R., TesSAdORI J., TAVERnA S., ChiAPPALONE M., «In vitro large-scale experimental and theoretical studies for the realization of bi-directional brain-prostheses ", Frontiers in Neural Circuits, 7:40, March 2013

[BRO14] BROWN, T., «On the nature of the fundamental activity of the nervous centres; together with an analysis of the conditioning of rhythmic activity in progression and a theory of the evolution of function in the nervous system», $\mathrm{J}$. Physiol.48, 18-46, 1914.

[CAP16] CAPOGROSSO M., ET AL., «A brain-spine interface alleviating gait deficits after spinal cord injury in primates», Nature, 539, 284-288, November 2016

[CAS08] CASSIDY, A., AND ANDREOU, A. G., «Dynamical digital silicon neurons» dans IEEE Biomedical Circuits and Systems Conference, BioCAS 2008,289-292. doi:10.1109/BIOCAS.2008.4696931, 2008.

[CAZ95] CAZALETS, J. R., BORDE, M., AND CLARAC, F., «Localization and organization of the central pattern generator for hindlimb locomotion in newborn rat», J. Neurosci. 15, 4943-4951., 1995. 
[CHA10] Charvet, G., Rousseau, L., Billoint, O., Gharbi, S., Rostaing, J. P., Joucla, S., ET Al., «BioMEA: A versatile high-density 3D microelectrode array system using integrated electronics. Biosens», Bioelectron. 25, 18891896. doi: 10.1016/j.bios.2010.01.001, 2010.

[COH92] Cohen, A. H., Ermentrout, G. B., Kiemel, T., Kopel, N., Sigvardt, K. A., And Williams, T. L., «Modelling of intersegmental coordination in the lamprey central pattern generator for locomotion», Trends Neuros $\mathrm{c}$ i.15, 434-438. doi:10.1016/0166-2236(92)90006-T, 1992.

[CRE06] CRESPI, A. AND IJSPEERT, A. J., «AmphiBot II : An amphibious snake robot that crawls and swims using a central pattern generator»,. dans Proceedings of the 9th international conference on climbing and walking robots (CLAWAR 2006), number BIOROB-CONF-2006-001, pages 19-27., 2006.

[CYM02] Cymbalyuk, G. S., Gaudry, Q., Masino, M. A., And Calabrese, R. L., «Bursting in leech heart interneurons: cell-autonomous and network-based mechanisms», J. Neurosci.22, 10580-10592, 2002.

[EKE93] EKEBERG, O., «A combined neuronal and mechanical model of fish swimming», Biological cybernetics, 69(5-6) :363-374, 1993.

[FIT55] FITZHugh, R., «Mathematical models of threshold phenomena in the nerve membrane», The bulletin of mathematical biophysics, 17(4) :257-278., 1955.

[FIT61] FitzHugh, R., «Impulses and physiological states in theoretical models of nerve membrane», Biophysical journal, 1(6) :445., 1961.

[Gue06] Guevremont, L., Renzi, C. G., Norton, J. A., Kowalczewski, J., Saigal, R., and Mushahwar, V. K., «Locomotor-related networks in the lumbosacral enlargement of the adult spinal cat: activation through intraspinal microstimulation», IEEE Trans. Neural Syst. Rehabil. Eng. 14, 266-272. doi: 10.1109/TNSRE.2006.881592., 2006.

[GRA11] Grassia, F., BuHry, L., LeVI, T., TOMAS, J., DesteXhe, A., AND SAIGHI, S. «Tunable neuromimetic integrated system for emulating cortical neuron models». Front. Neurosci.5:134. doi: 10.3389/fnins.2011.00134, 2011.

[GRA17] Grassia F., KoHNo T., LEVI T., « Digital hardware implementation of a stochastic two-dimensional neuron model », Journal of Physiology-Paris, February 2017

[Hei12] Heim, M., Rousseau, L., Reculusa, S., Urbanova, V., Mazzocco, C., Joucla, S., et Al., «Combined macro/mesoporous microelectrode arrays (MEAs) for low noise extracellular recording of neural networks». J. Neurophysiol. 108, 1793-1803 doi: 10.1152/jn.00711.2011, 2012.

[HIL01] Hill, A. A., Lu, J., MAsino, M. A., Olsen, O. H., AND CAlABRESE, R. L., «A model of a segmental oscillator in the leech heartbeat neuronal network», J. Comput.Neurosci.10, 281-302. doi: 10.1023/A:1011216131638, 2001.

[HOC06] Hochberg, L. R., Serruya, M. D., Friehs, G. M., Mukand, J. A., Saleh, M., Caplan, A. H., et al., «Neuronal ensemble control of prosthetic devices by a human with tetraplegia», Nature442, 164-171. doi: 10.1038/nature04970, 2006.

[HOC12] Hochberg, L. R., Bacher, D., Jarosiewicz, B., MASSe, N. Y., Simeral, J. D., Vogel, J., Haddadin, S., LiU, J., CASH, S. S., VAN DER SMAGT, P., AND DONOGHUe, J. P., «Reach and grasp by people with tetraplegia using a neurally controlled robotic arm», Nature, 485(7398) :372-375, 2012.

[HOD52] HodgKIN, A. L., AND HuXLEY, A. F., «A quantitative description of membrane current and its applications to conduction and excitation in nerve», J. Physiol. 117, 500-544., 1952.

[HOO00] HOOPER, S., «Central pattern generators», Curr. Biol.10, 176-177. doi: 10.1016/S0960-9822(00)00367-5, 2000.

[IJS01] IJSPEERT, A., «A connectionist central pattern generator for the aquatic and terrestrial gaits of a simulated salamander», J. Biol. Cybern. 84, 331-348. doi:10.1007/s004220000211, 2001.

[IJS07] IJSPEERT, A., CRESPI, A., RYCZKO, D., AND CABELGUEN, J., «From swimming to walking with a salamander robot driven by a spinal cord model», Science315, 1416-1420. doi: 10.1126/science.1138353, 2007.

[IJS08] IJSPEERT, A., «Central pattern generators for locomotion control in animals and robots: a review», J. Neural Netw.21, 642-653. doi: 10.1016/j.neunet.2008. 03.014, 2008.

[IZH03] IZHIKEVICH, E. M.., «Simple model of spiking neurons», IEEE Trans. Neural Netw.14, 1569-1572. doi: 10.1109/TNN.2003.820440, 2003.

[IZH04] IZHIKEVICH, E. M., «Which model to use for cortical spiking neurons», IEEE Trans.NeuralNetw.15, 1063-1070. doi: 10.1109/TNN.2004.832719, 2004. 
[JOU16] Joucla, S., Ambroise, M., LeVI, T., LAFon, T., Chauvet, P., SAÏGHI, S., Bornat, Y., Lewis, R., RenAud, S. AND YVERT, B., «Generation of locomotor-like activity in the isolated rat spinal cord using intraspinal electrical microstimulation driven by a digital neuromorphic CPG», Frontiers in neuroscience, 10, 2016.

[JUN01] JUNG, R., BRAUER, E. J., AND ABBAS, J. J., «Real-time interaction between a neuromorphic electronic circuit and the spinal cord», IEEE Trans. Neural Syst. Rehabil. Eng.9, 319-326. doi: 10.1109/7333.948461, 2001.

[KIE03] KIEHN, O., AND BUTT, S. J. «Physiological, anatomical and genetic identification of CPG neurons in the developing mammalian spinal cord», Prog. Neurobiol. 70, 347-361. doi: 10.1016/S0301-0082(03)00091-1, 2003.

[LEM02] Le Masson, G., Renaud-Le Masson, S., Debay, D., And Bal, T., «Feedback inhibition controls spike transfer in hybrid thalamic circuits», Nature417, 854-858. doi: 10.1038/nature00825., 2002.

[LEV08] LeVi T., Lewis N., SAighi S., TOMas J., Bornat Y., RenAud S., « Neuromimetic Integrated Circuits », Chap. 12 in VLSI Circuits for Biomedical Applications, Artech House, Boston, pp. 241-264, 2008

[LEV16] LEVI T AND FUJII T, «Microfluidic neurons: a new way in neuromorphic engineering? », Micromachines, 7:146, August 2016

[LEV17] LeVi T, Khoyratee F, SAighi S, IKeUChi Y, « Digital implementation of Hodgkin-Huxley neuron model for neurological diseases studies », Journal on Artificial Life and Robotics, 1-5, September 2017

[LI13] LI, C. A. I., LOWE, R., AND ZIEMKE, T., «Humanoids Learning to Walk : a Natural CPG-Actor-Critic Architecture», Frontiers in Neurorobotics, 7(5)., 2013.

[LI14] LI, W.-C., MERRISON-HORT, R., ZHANG, H.-Y., AND BORISYUK, R., «The generation of antiphase oscillations and synchrony by a rebound-based vertebrate central pattern generator», The Journal of Neuroscience, 34(17) :60656077., 2014.

[MAR01] MARDER, E., AND BUCHER, D. «Central pattern generators and the control of rhythmic movements. Curr. Biol.11, 986-996. doi: 10.1016/S0960-9822(01)00581-4, 2001.

[MAT87] MATSUOKA, K., «Mechanism of frequency and pattern control in the neural rhythm generators», Biol. Cybern.56, 345-353. doi: 10.1007/BF00319514, 1987.

[MAZ12] MazureK, K. A., Holinski, B. J., Everaert, D. G., Stein, R. B., Etienne-Cummings, R., And MushahWAR, V. K., «Feed forward and feedback control for over-ground locomotion in anaesthetized cats», J. Neural Eng. 9, 26003. doi : 10.1088/1741-2560/9/2/026003, 2012.

[NAG62] NAgUmo, J., ARIMOTO, S., AND Yoshizawa, S., «An active pulse transmission line simulating nerve axon», Proceedings of the IRE, 50(10):2061-2070., 1962.

[NIC09] NiCOlelis, M. A. L. AND LeBedeV, M. A., «Principles of neural ensemble physiology underlying the operation of brain-machine interfaces», Nat Rev Neurosci, 10(7) :530-540., 2009.

[Sha14] Shahdoost, S., Frost, S., Acker, G., Van Dejong, S., Barbay, S., Nudo, R., Et Al., «Towards a miniaturized brain-machine-spinal cord interface (bmsi) for restoration of function after spinal cord injury», in 36th Annual International Conference of the IEEE Engineering in Medicince and Biology Society (Chicago, IL), 486-489, 2014.

[STE87] STEVEnSON, P. A. AND KUTSCH, W., «A reconsideration of the central pattern generator concept for locust flight», Journal of Comparative Physiology A, 161(1) :115-129., 1987.

[STE67] STEIN, R. B., «Some models of neuronal variability», Biophysical journal, 7(1) : 37., 1967

[VAN20] VAN DER POL, B., «A theory of the amplitude of free and forced triode vibrations», Radio Review, 1(1920) : 701-710., 1920.

[VAN28] VAN DER POL, B., «The heartbeat considered as a relaxation oscillation, and an electrical model of the heart», Philos. Mag.6, 763-775., 1928.

[VOG06] Vogelstein, R. J., Tenore, F., Etienne-Cummings, R., Lewis, M. A., And Cohen, A. H., «Dynamic control of the central pattern generator for locomotion», Biol. Cybern.95, 555-566. doi: 10.1007/s00422-006-0119-z, 2006.

[ZIE96] ZIELINSKA, T. «Coupled oscillators utilised as gait rhythm generators of a two-legged walking machine», Biological Cybernetics, 74(3) : 263-273., 1996. 